\title{
ADVANCED METHODS OF POTATO LOSS REDUCTION IN STORAGE
}

\author{
Alexander Lysakov, Gennady Nikitenko, Evgeny Konoplev, Yaroslav Tarasov \\ Stavropol State Agrarian University, Russia \\ s_lysakov@mail.ru, nikitenko_gv@mail.ru, konoplev82@mail.ru, tarasov-yar@mail.ru
}

\begin{abstract}
Modern techniques of potato tubers storage have a number of economic, technical, and technological disadvantages and they do not ensure high product safety - potato losses run up to $30 \%$ per storage season. The main goal of the experiment conducted by the authors is a research investigation of the impact made by electrophysical methods on reservation capacity of potato tubers, because this method is the most interesting and understudied. During the experimental studies potato tubers were exposed to electromagnetic direct-current field, electromagnetic alternating current field, ionization, microwave field. The equipment used in the research: equipment for magnetic potato treatment, a microwave field generator, a generator of air ions, DC and AC voltage sources, and auxiliary instruments. As a result of the experimental studies it has been found that untreated potatoes had mass loss of $39 \%$. Mass loss of potatoes treated with microwave field was over $50 \%$. Potatoes exposed to aeroionization lost $23 \%$ of their mass. At a magnetic treatment dose of 6-8 mT.s mass loss of potatoes does not exceed $20 \%$, but when a dose of magnetic treatment is greater or less than the specified ranges, mass loss of potatoes exceeds $40 \%$ reaching $75 \%$. Hence it has been demonstrated that the method of electromagnetic treatment within specific ranges is the most economical one. According to the results of the experiments, electromagnetic potato tubers treatment at a dose of 6-9 $\mathrm{mT} \cdot \mathrm{s}$ has been recognized as the most effective option.
\end{abstract}

Keywords: food loss, ionization, microwave field, magnetic field.

\section{Introduction}

Proper storage conditions of agricultural products make possible a year-round supply of food retaining its high nutritional and taste qualities and habits. In addition, proper storage of agricultural products is closely linked to the food security of the country, therefore, improvement of storage conditions and loss reduction in the potato warehouses are important and urgent problems [1].

A forced blowing method, use of chemical agents-inhibitors, obtaining genetically-modified (GM) potato, which does not rot, are the main modern potato storage methods. However, for a variety of reasons, these methods have several disadvantages of economic, technical, and technological nature. For example, the forced blowing method is the most energy-consuming one, as it requires a large number of sensors, motors, heating and cooling systems, which must work practically day-andnight for the entire duration of a storage period. Potato warehouses are often fitted with outdated inefficient ventilation equipment. The increase in electricity and fuel prices force to saving; it leads to limitation of electrical equipment operation at the potato warehouses; in its turn it has an adverse effect on the storage process and the quality of the stored products. As a result we have increase in potato losses; suppliers try to recoup their losses and raise the prices [2].

The second most common technique of storage and loss reduction is treatment of potato tubers with biological and chemical protective-stimulating agents and germination inhibitors before storing. Tubers treatment with germination inhibitors of different chemical compositions containing chlorpropham as an active substance is required in most foreign techniques. Depending on a kind of drugs (powder or fluid) tubers are treated before or during storing (if an inhibitor is in the form of smoke cartridge). This method significantly reduces losses caused by potato mass loss (up to $12 \%$ ); and it is very often used in conjunction with a forced blowing method. However, it should be noted that not all kinds of potato have similar reaction to inhibitor treatment. In addition, the inhibitors contain chlorine in their composition. Chlorine accumulated in products can be harmful to people [3; 4].

The third way of potato storage is the use of genetically-modified potato; it enables to reduce losses caused by diseases and decay to almost $0.5 \%$. Genetically-modified potato is not subjected to diseases; it does not rot and can be stored for several years. However, research on the impact of GM foods on humans and animals gives contradicting results; so the international community is not ready to use GM foods. 
The main goal of the experiment conducted by the authors is a research investigation of the impact made by electrophysical methods on reservation capacity of potato tubers because this method is the most interesting and understudied.

\section{Materials and methods}

Modern research made it possible to achieve significant progress in the organization of potato storage, but the losses still remain quite high and the tuber quality during holding is significantly spoiled; that is why attempts to use other methods of potato tubers treatment before storing are made. These methods are electrophysical: application of electromagnetic fields (constant, alternating, pulsating), microwave fields, the use of negative ions, electric fields of the corona discharge [5-7]

The authors studied the following physiological factors: treatment of potato tubers with constant electromagnetic field, alternating electromagnetic field, ionization of potato, and potato treatment with microwave field. During the experiment, the following tasks were completed: determination of the nature of electrophysical action on potato tubers; setting optimal parameters of electrophysical action on reduction of potato mass loss; determination of the mass variation nature of treated potato tubers mass compared with untreated.

During the experiment standard the following research methods were applied: a multifactorial experiment method, a statistical analysis, an adequacy test. All measuring instruments used in the experiment are certified in the Russian Federation; they include: electronic scales, a voltmeter, an amperemeter, a professional stopwatch, a milliteslameter, a pyrometer for temperature measurement, humidity sensors, a microscope, and an ion counter [8; 9]. A cultivar of potato used in all the experiments is "Aurora". Treatment efficiency was assessed by the remaining mass of the tubers calculated by the formula:

$$
\Delta=100-\left(\frac{m_{E N D}}{m_{B E G}} \cdot 100\right), \%
$$

where $\quad m_{E N D},-$ weight of tubers at the end of the experiment, gram;

$m_{B E G}-$ weight of tubers at the beginning of the experiment, gram.

During the experimental studies unharmed and harmed tubers were exposed to electrophysical treatment and were laid for storing in packages for 16 days at constant air temperature $25^{\circ} \mathrm{C}$ and $60 \%$ of humidity. These storage parameters were chosen in order to accelerate the processes of decay and potato sprouting. Daily throughout the experiment the weight of all tubers, area and thickness of protective surface of harmed tubers were measured; decay surface area of harmed tubers and its changes were also measured.

Treatment of potato tubers with negative ions has been done as follows: potatoes were put in a closed container and a generator of negative ions ("Ovion"- consumer grade) was set. The generator was turned on for a time from $60 \mathrm{~s}$ to $900 \mathrm{~s}$; the ion concentration was 1900 ions $\cdot \mathrm{cm}^{-3}$.

A significant number of experimental studies are associated with the pre-sowing electromagnetic treatment of plants. Similar research was conducted everywhere: in Russia, the USA, China, the EU, etc. Studies on the spark discharge treatment, ultrasound, ozone treatment, X-ray irradiation were also carried out $[10 ; 11]$. All these methods are energy consuming compared to electromagnetic treatment. However, there is almost no research on electromagnetic food treatment before storing. Electromagnetic method of treatment is the most economical and environmentally friendly, so that magnetic treatment devices of different constructions and for various purposes in the pre-experimental studies were applied.

Currently, the design of the machine for magnetic treatment is being implemented with the help of software products, which allow us to simulate all processes taking place inside the machine, to optimize the device design, to simplify the design, and check productivity of any circuit of the electromagnetic treatment device. Based on the results of mathematical engineering the machine design optimally suitable for the potato treatment has been developed [8].

The electromagnetic machine has a completely new design, which gives an opportunity to change both the magnetic treatment process and means of its implementation in positive manner. Design and 
manufacturing technology of this machine make it possible to adapt it for a specific task of magnetic treatment, to use machines of improved performance with minimum energy consumption.

The electromagnetic machine for potato tubers treatment, which diagram is represented in Figure 1 , consists of a hopper 1, made of nonmagnetic material; potatoes 2 are loaded in a hopper $1\left(Q_{3}\right)$; a pipeline 3 having a work area for processing. A source of electromagnetic field is composed of the magnetizing coils 4 and 7, set one by one along the pipe 3 and enclosed in the magnetic cores 5 . Connection of magnetizing coils 4 and 7 is consistent. Magnetic paths 5 are designed to improve the efficiency of electromagnetic treatment by loss reduction of electromagnetic energy dispersion in the environment. A non-magnetic guide spiral 6 is designed for electromagnetic treatment of potato tubers 2. Discharge is made through the unloading hopper $8\left(Q_{b}\right)$. Hoppers 1 and 8 , a pipeline 3 and magnetic paths 5 are fixed by welding.

When voltage is supplied from a current source, the current flowing through the magnetizing coils causes the magnetic fluxes $\Phi 1, \Phi 2, \Phi 3, \Phi 4$. Magnetic fluxes $\Phi 1, \Phi 2, \Phi 3, \Phi 4$ are workflows that have impact on the object to be processed, which is a potato tuber.
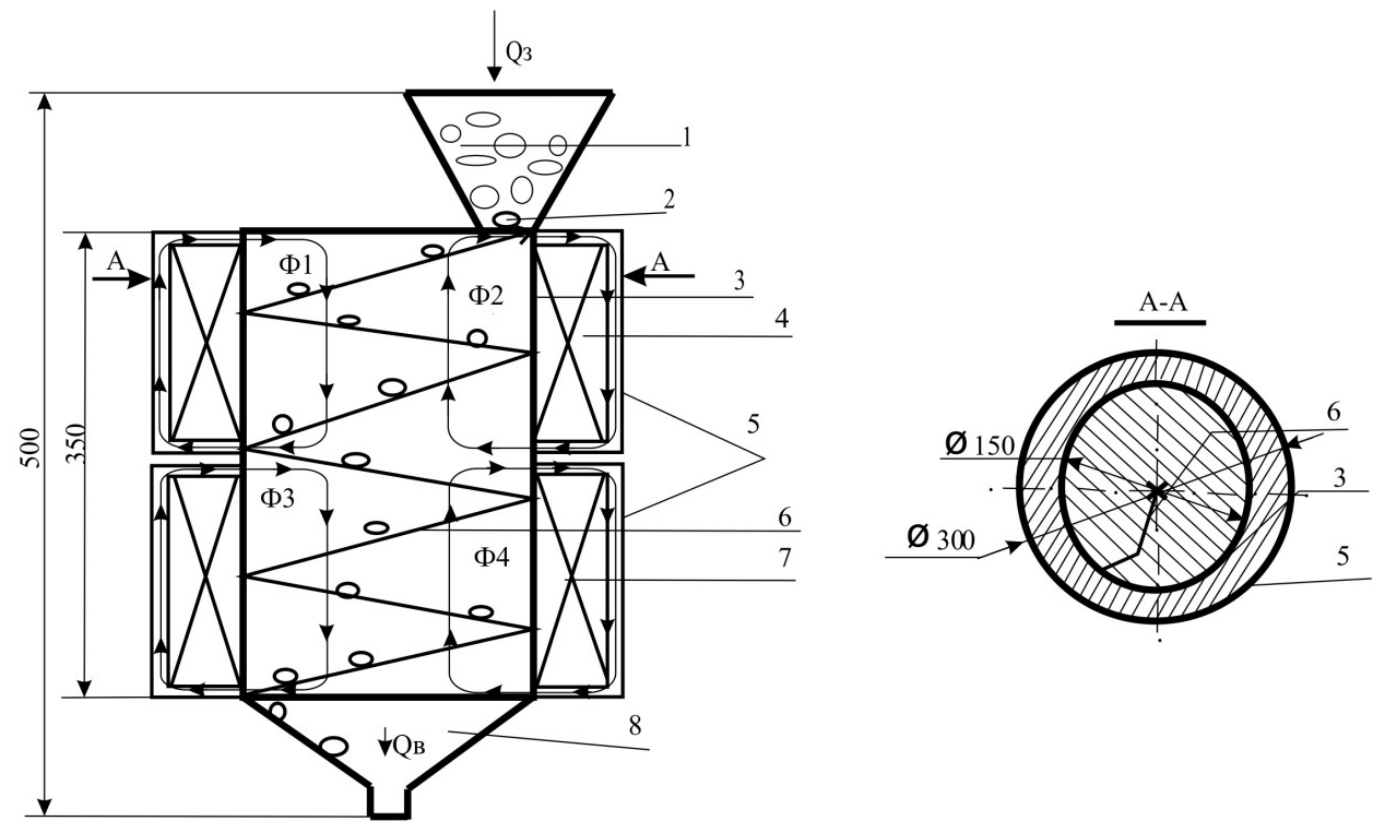

Fig. 1. General form and sectional drawing of machine for electromagnetic treatment of potato tubers

The experimental machine for electromagnetic treatment of potato tubers was designed according to standard parameters of electric network: $220 \mathrm{~V}$ and frequency $50 \mathrm{~Hz}$. When the machine is connected to AC, magnetic induction in the treatment area is $0.35 \mathrm{mT}$. When using AC-to-DC conversion, the voltage value remained constant and was equal to $220 \mathrm{~V}$. Magnetic induction in the treatment zone at a constant current was $1.27 \mathrm{mT}$.

For the microwave treatment an experimental stand-simulator on basis of a household microwave oven was applied. During the experiment, potato tubers were treated with microwave field at frequency $2500 \mathrm{MHz}$, treatment time varied from 1 to $30 \mathrm{~s}$.

\section{Results and discussion}

The experimental results obtained in the process of potato tubers treatment with microwave fields have demonstrated that the minimum mass loss of $39 \%$ was observed in the control group; even initial treatment for $1 \mathrm{~s}$ decreases the potato mass to $50 \%$ over 16 days. This treatment method is appropriate for dehumidification or other heat treatment for further processing (Fig. 2). In particular, there is a storage technology for chips potato, when the tubers are subjected to thermal heating $[3 ; 5 ; 7]$. 


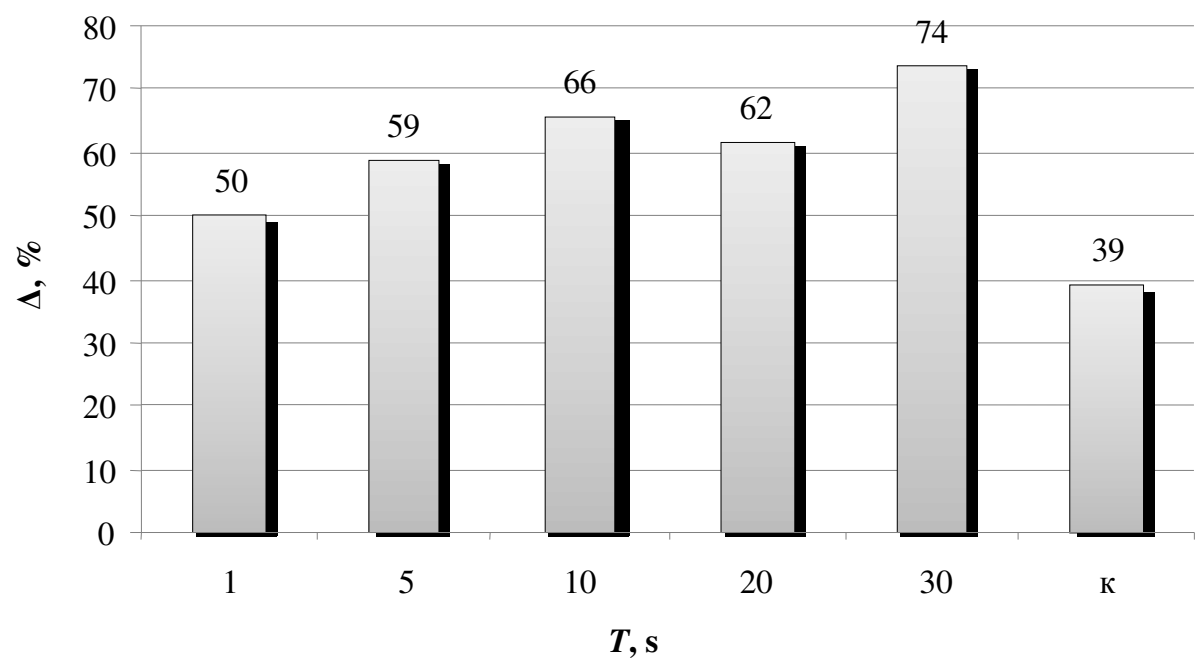

Fig. 2. Dependence of potato mass loss $\Delta(\%)$ on time of microwave treatment $T, \mathrm{~s}(\kappa$ - untreated control)

The results of experimental studies on the treatment of potato tubers with negative air ions, shown in Figure 3, have demonstrated that the greatest mass loss (39\%) is observed in the control group, but increase of the treatment time with aero ions does not reduce the mass loss, as expected in the course of the experiment, and it is unstable. Optimal treatment regimen is $300 \mathrm{~s}$ or close to it, at a constant ion concentration 1900 ions $\cdot \mathrm{cm}^{-3}$.

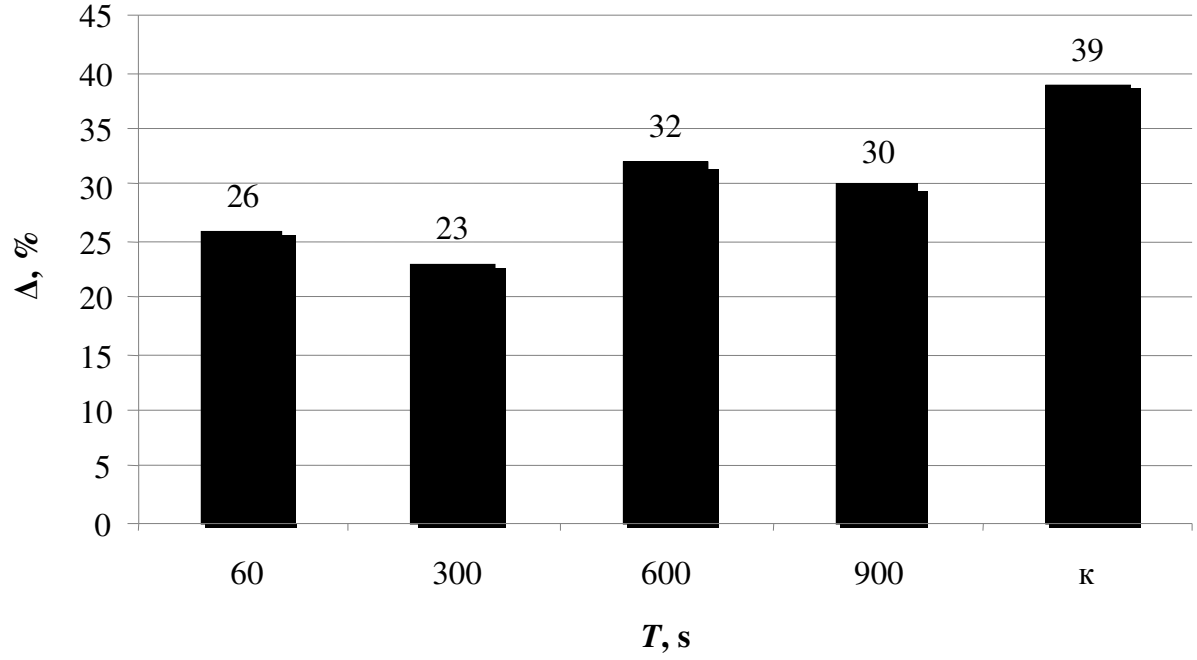

Fig. 3. Dependence of potato mass loss $\Delta(\%)$ on time of negative air ions treatment $T, \mathrm{~s}(\kappa-$ untreated control)

Potato treatment with the electromagnetic field was divided in accordance with the current type used. One part was treated with DC electromagnetic field at induction of $1.27 \mathrm{mT}$ and the treatment time from 10 to $60 \mathrm{~s}$ in increments of $10 \mathrm{~s}$. Another part was treated with AC electromagnetic field at induction of $0.35 \mathrm{mT}$ and the same time ranges. Tubers treatment and tubers storage were conducted under conditions identical to the both parts and the untreated control. Table 1 shows the values of magnetic induction $(B)$ and time periods, at which the maximum and minimum weight losses were observed; the intermediate results are not shown.

In the result of the study, it has been found that the minimal weight loss was observed after treatment with DC electromagnetic field for experience No. 1 (Table 1).

It has been found that during potato tubers treatment with alternating electromagnetic field a treatment dose plays an important role. It is defined as multiplication of magnetic induction $B$ (Tesla) by time period $t(\mathrm{~s})$ of the object in a magnetic field. During the experiment, the dose of magnetic treatment is within the range from $5 \mathrm{mT} \cdot \mathrm{s}$ up to $15 \mathrm{mT} \cdot \mathrm{s}$. 
Results of electromagnetic potato treatment

Table 1

\begin{tabular}{|c|c|c|}
\hline No. experience & Type and treatment parameters & Mass loss, \% \\
\hline 1 & $\begin{array}{c}\text { DC electromagnetic field } \\
B=1.27 \text { mT, treatment time } 10 \mathrm{~s}\end{array}$ & 18 \\
\hline 2 & $\begin{array}{c}\text { DC electromagnetic field } \\
B=1.27 \text { mT, treatment time } 60 \mathrm{~s}\end{array}$ & 61 \\
\hline 3 & $\begin{array}{c}\text { AC electromagnetic field } \\
B=0.35 \mathrm{mT} \text {, treatment time } 20 \mathrm{~s}\end{array}$ & 60 \\
\hline 4 & $\begin{array}{c}\text { AC electromagnetic field } \\
B=0.35 \mathrm{mT} \text {, treatment time } 60 \mathrm{~s}\end{array}$ & 72 \\
\hline 5 & The control (untreated potato) & 39 \\
\hline
\end{tabular}

Figure 4 shows the average data on dependence of potato tubers mass loss on the dose of magnetic treatment and the duration of the experimental studies.

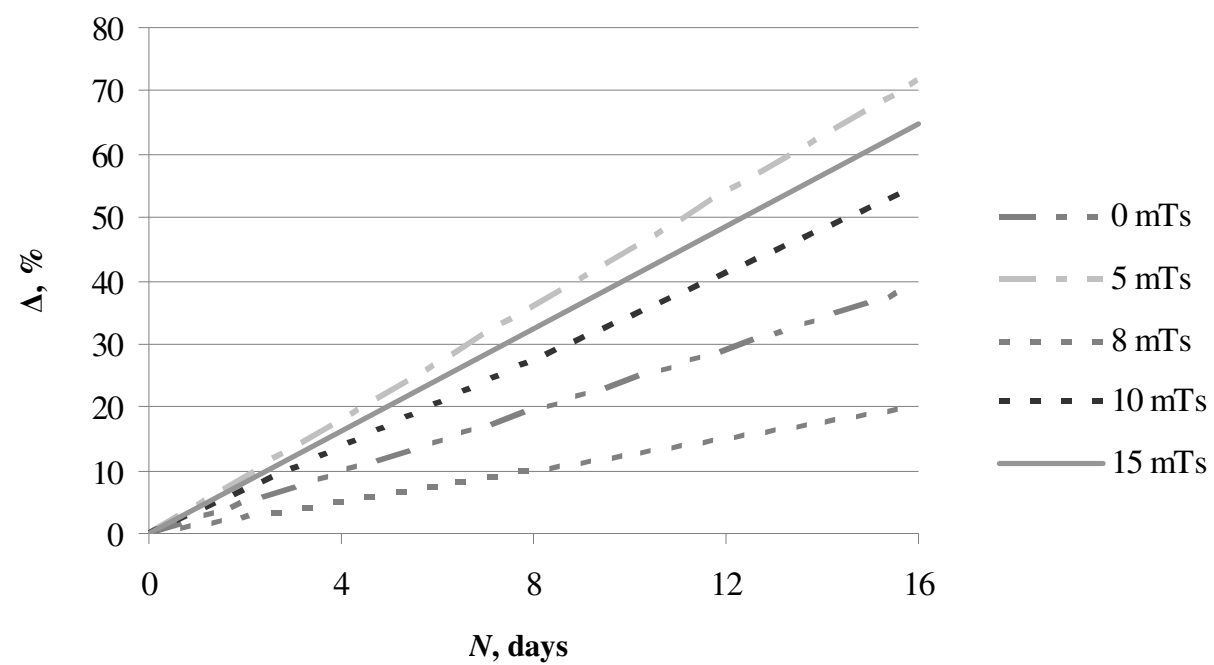

Fig. 4. Dependence of potato tubers mass loss $\Delta(\%)$ on dose of magnetic treatment $B \cdot t(\mathrm{mT} \cdot \mathbf{s})$ and duration of experimental studies $N$ (days)

The graph shows that the smallest mass loss is observed in options 1 and 3 , that corresponds to the untreated control, and potatoes treated at a dose of magnetic field $B \cdot t=8 \mathrm{mT} \cdot \mathrm{s}$. The other options have very high mass loss over $50 \%$. According to the authors, within the range $B \cdot t=7 \ldots 9 \mathrm{mT} \cdot \mathrm{s}$ additional studies are required.

During the experiment it is determined that mechanical damage (wounds) of the treated potato tubers are tightened (healed) more quickly due to the increase in the speed of potato tissue formation, and moreover, it occurs at the surface of the protective cover. It is also found that the parameters of the electromagnetic field within the range of $B \cdot t=7 \ldots 9 \mathrm{mT} \cdot \mathrm{s}$ have depressing influence on fungi and bacteria, which are the cause of buck eye rot, Phoma rot, Fusarium blight, Fusarium, rubbery rot and other diseases. It leads to the improvement of storage conditions in the environment with high-intense heat $\left(25-28{ }^{\circ} \mathrm{C}\right)$, where potato tubers are not exposed to rotting and keep their food properties. Parameters of electromagnetic field within the range of $B \cdot t=13 \ldots 15 \mathrm{mT} \cdot \mathrm{s}$ conversely have a positive effect on emergence and development of fungi and bacteria, and lead to increase in their number and as a consequence we observed accelerated decay process and sharp loss of potato tubers useful mass.

\section{Conclusions}

1. Application of electrophysical modality on potato is a controversial issue and enables both to slow down the process of mass loss and potato rotting, and speed up the process. 
2. As a result of the experimental studies it has been found that untreated potatoes had mass loss of $39 \%$. Mass loss of potatoes treated with microwave field was over $50 \%$. Potatoes exposed to aeroionization lost $23 \%$ of their mass.

3. Results of potato tubers treatment with electromagnetic field allow us to conclude that there is a value (or range of values) for a dose of magnetic treatment, when mass losses of treated potato are less than in the untreated control group. At a magnetic treatment dose of 6-8 mT.s mass loss of potatoes does not exceed $20 \%$, but when a dose of magnetic treatment is greater or less than the specified ranges, mass loss of potatoes exceeds $40 \%$ reaching $75 \%$.

4. According to the results of the experiments, electromagnetic potato tubers treatment at a dose of 6-9 $\mathrm{mT} \cdot \mathrm{s}$ has been recognized as the most effective option.

5. Preliminary calculations have shown that production losses can be reduced from $30 \%$ to $5 \%$ by introduction of electromagnetic treatment in the technology for long-time potato storage; it is recommended in conjunction with the forced blowing method.

\section{References}

[1] Beretta C., Stoessel F., Baier U., Hellweg S. Quantifying food losses and the potential for reduction in Switzerland. Waste Management, vol. 33 (3), 2013, pp. 764-773.

[2] Mehta A., Singh B., Ezekiel R., Minhas J.S. Processing quality comparisons in potatoes stored under refrigerated and non-refrigerated conditions. Indian Journal of Plant Physiology, vol. 19 (2), 2014, pp. 149-155.

[3] Driskill Jr. E.P., Knowles L.O., Knowles N.R. Temperature-induced changes in potato processing quality during storage are modulated by tuber maturity. American Journal of Potato Research, vol. 84 (5), 2007, pp. 367-383.

[4] Mehta A., Ezekiel R. Potato storage: Need, present scenario, emerging technologies and future strategies: A critical appraisal. Journal of Food Science and Technology, vol. 43 (5), 2006, pp. 453-466.

[5] Mahto R., Das M. Effect of $\gamma$ irradiation on the physico-mechanical and chemical properties of potato (Solanum tuberosum L), cv. 'Kufri Chandramukhi' and 'Kufri Jyoti', during storage at $12^{\circ} \mathrm{C}$. Radiation Physics and Chemistry, vol. 107, 2014, pp. 12-18.

[6] Mahto, R., Das, M. Effect of gamma irradiation on the physico-mechanical and chemical properties of potato (Solanum tuberosum L.), cv. 'Kufri Sindhuri', in non-refrigerated storage conditions. Postharvest Biology and Technology, vol. 92, 2014, pp. 37-45.

[7] Frazier M.J., Kleinkopf G.E., Brey R.R., Olsen N.L. Potato sprout inhibition and tuber quality after treatment with high-energy ionizing radiation. American Journal of Potato Research, vol. 83 (1), 2006, pp. 31-39

[8] Grinchenko V., Nikitenko G., Lysakov A., Konoplev E. Milking machine with electropulsator. Proceedings of International conference «Engineering for Rural Development», 2016, Latvia University of Agriculture, pp. 230-235.

[9] Grinchenko V., Nikitenko G., Lysakov A., Konoplev E. Duration of change of pressure in interwall camera of milking cup. Proceedings of International conference «Engineering for Rural Development», 2017, Latvia University of Agriculture, pp. 819-823.

[10] Kumar S., Petwal V.C., Kaul A., Behere A., Promod R., Bapna S.C., Soni H.C., Sharma A. Sprout inhibition in potato (Solanum tuberosum L.) with low energy electrons. Journal of Food Science and Technology, vol. 46 (1), 2009, pp. 50-53.

[11] Blessington T., Scheuring D., Nzaramba M., Hale A., Reddivari L., Vestal T., Maxim J., Miller Jr. J., The Use of Low-Dose Electron-Beam Irradiation and Storage Conditions for Sprout Control and their Effects on Xanthophylls, Antioxidant Capacity, and Phenolics in the Potato Cultivar Atlantic. American Journal of Potato Research, Volume 92, Issue 5, 2015, pp. 609-618 